\title{
XXXVII.
}

\section{Ein Beitrag zur Symptomatologie cerebraler Hemiplegieen.}

\author{
Von \\ Dr. Ottomar Rosenbach, \\ Assistenzarzt an der medicinischen Klinik zu Jena.
}

Durch eine interessante Mittheilung ron Jastrowitz*) ist die Aufmerksamkeit auf ein Symptom cerebraler Hemiplegieen bei Männern gelenkt worden, welches in theoretischer und practischer Beziehung eine eingehende Berücksichtigung verdienen dürfte; es ist dies das Verhalten des Cremaster bei halbseitigen Lähmungen in Folge von Gehirnaffectionen (Hämorrhagien oder Embolieen). Im Anschluss an die Schilderung des verschiedenen Verhaltens des genannten Muskels auf der gesunden Seite gegenüber der erkrankten, - Ausbleiben des reflectorischen Emporziehens des Hodens auf der letzteren - hat Jastrowitz die Frage von dem Verhalten der Rumpfmusculatur einer Erörterung unterzogen und auf die Bedeutung seines Symptomes für die Annahme einer Lähmung der Bauchousculatur hingewiesen. Meine Mittheilung beabsichtigt sowohl einen kleinen Beitrag zur Lösung dieser Frage zu liefern, als auch auf ein Symptom hinzuweisen, welches bei seiner Unabhängigkeit von dem Geschlecht der Kranken wohl geeignet ist, in zweifelhaften Fällen zur Diagnose des Sitzes der Erkrankung benutzt zu werden. Abgesehen von dem practischen Werthe des Zeichens, hat dasselbe auch in theoretischer Beziehung ein gewisses Interesse, da es auf dem Ausbleiben eines in normalen Verhältnissen leicht hervorzurufenden Reflexes bernht, also von Wichtigkeit für die Theorie der Reflexhemmung von Seiten des Gehirns ist. Eine Erklärung des Phänomens nach dieser letzteren Richtung hin beabsichtige ich nicht, da die Klarstellung nach dieser Seite hin entweder einer experimentellen Arbeit bedarf (wie schon Jastrowitz bemerkt hat), eine Arbeit, die ich erst begonnen habe, oder wenigstens einer Reihe unzweifelhafter Obductionsergebnisse, über die ich noch nicht verfüge. An diesem Orte habe ich nur vor, die Resultate meiner Beobachtungen an einer Reihe von Hemiplegieen, die ich im Laufe von 1//2 Jahren Gelegenheit hatte zu sehen, zu publiciren.

*) Berliner klin. Wochenschrift. 1875, S. 428 . 
Da in allen frischen Fällen das bald zu beschreibende Zeichen nicht im Stiche liess, da selbst in den älteren Fällen ziemlich prägnante Resultate sich ergaben, so glaube ich diese Mittheilung wohl wagen zu durfen, und die Anwendung des in Rede stehenden Zeichens empfehlen zu können.

Es ist eine bekannte Thatsache, dass man bei plötzlichen Berührungen des Abdomens, bei Streichen desselben, ja bei blossen Berührungen der Bauchwand reflectorische Finziehungen derselben bewirken kann. Diese auf Contraction der Bauchmusculatur beruhenden Einziehungen können halbseitig, wenn der Hautreiz die Mittellinie nicht überschreitet, hervorgerufen werden. Am deutlichston tritt das Einsinken anf, wenn man mit dem Nagel schnell in grösserer oder geringerer Ausdehnung übrr die Bauchfäche fährt, und zwar ist die Richtung des Striches meist indifferent. Bisweilen erbält man starke einseitige Einziehung des Epigastrium bei transversalem Streichen desselben (äusserst selten eine doppelseitige Einziebung); bisweilen ist die Einziehung stärker bei Streichen längs oder parallel der Mittellinie; oft wirken schräge Striche am besten. Namentlich dentlich tritt hier oft ein seitliches Verziehen des Nabels ein. Sehr schön sieht man oft die Contraction der Musculatur in grosser Ausdehnung bei leisem Ueberfahren des Leistencanals. In seltenen Fällen ist das Zeichen an der Vorderfläche nicht zu eruiren, dann aber meist in der Seitenwand. Selbst durch Ueberfabren der untersten Intercostalräume oder durch leichte Nadelstiche an einer Stelle des Abdomens kann man das Phänomen hervorrufen, nie aber bei Streichen des Schenkels unterbalb der Inguinalfurche.

Berährung mit kalten Gegenständen ist ebenfalls oft im Stande das Zeichen zu erzengen, am besten wirken jedoch die Striche mit dem Fingernagel. Wendet man das beschriehene Verfahren bei Hemiplegieen aus cerebraler Ursache an, so erfolgt auf der gelähmten Seite kein solcher Bauchreflex, wie ich das Phänomen nennen möchte, während auf der gesunden Seite derselbe unverändert und prompt eintritt. In frischeren Fïllen ist, wie oben erwähnt, das Versagen des Bauchreflexes stets zu constatiren, bei längerer Dauer der Läsion tritt er schwach auf, aber selbst bei sehr alten Fällen ist eine Differenz auf beiden Körperhälften zu constatiren, der Reflex bleibt, soweit ich dies beurtheilen kann, stets schwächer auf der erkrankten Seite. Individuen, bei denen eine Hyperalgesie auf der gelähmten Seite bestand, hatte ich nicht Gelegenheit zu untersuchen. (Beiläufig möchte ich hier erwähnen, dass ich nicht selten bei normalen Menschen ein schwächeres Auftreten des Reflexes auf der rechten Seite bemerkt habe, nie aber das umgekehrte. Ob diese Beobachtung nur zufälig ist, wage ich nicht $z u$ entscheiden.)

Was andere Krankheiten betrifft, so habe ich bei drei Fällen von Tabes in zwei Fällen einen auffallend starken, in einem Falle gar keinen Reflex auftreten sehen; bei essentieller Kinderlähmung und bei Hydrocephalus waren die Reflexe unverändert auf beiden Seiten vorhanden, ebenso in einem Falle von Compressionsmyelitis bei Spondylarthrocace. Unter einer grossen Reihe von untersuchten Fällen bei normalen oder nicht nervös erkrankten Individuen, sind mir nur zwei Fälle von Fehlen des Bauchreflexes vorgekummen; selbst wcnn er zu fehlen scheint, fördert eine Veränderung des Verfahrens in der oben beschriebenen Weise doch noch einen mehr oder minder deutlichen Reflex zu Tage. 
Ein zweites ebenfalls interessantes und, wie ich glaube, nicht unwichtiges Symptom bei Hemiplegieen ist das Verhalten der glatten Musculatur, die auch halbseitig afficirt zu sein scheint. Reizt man nämlich den Warzenhof der Brustdrüse bei Männern oder Frauen durch Streichen, so runzelt sich die Areola auf der gesunden Seito schnell und deutlich, und die Warze erigirt sich, während auf der gelähmten Seite dieser Vorgang entweder gar nicht eintritt, oder Iangsam und unvollkommen erfolgt. (Am Scrotum kommt ein ähnliches Phänomen vor, ist aber meist nicht so dentlich.)

In älteren Fällen ist diese Erscheinung nicht so prägnant. Vergleicht man die Areola der einen mit der anderen Seite, so fällt oft sofort die grössere Schlaffheit derselben, ihre glatte Beschaffenheit, gegenüber der mehr gerunzelten, einen kleinen Kreis darstellenden, auf der gesunden Seite auf, Es ist gut, die gesunde Seite zuerst za reizen, weil sich oft bei Reizung der einen Mamilla die andere mit contrahirt, man also, wenn man die Reaction der kranken Seite zuerst prüft, den Unterschied zwischen beiden in der Reaction nicht mehr so deutlich wahrnehmen kann als vorher.

Ich erlaube mir nun die von mir beobachteten Fälle kurz mit ihren Hauptsymptomen anzuführen.

1) Hämorrhag. cerebri. sin.

H. C., 75 Jahr alt, weiblich. Seit einem Jahre (1874) vollständige rechtsseitige Hemiplegie (Gesicht und Extromitäten). Bauchreflex fehlt rechts, links deutlich. Nach noch 1 Jahre (Januar 1876) hat sich die Lähmung ein wenig gebessert. Bauchreflex fehlt rechts, links sehr schwach. Mamilla rechts nicht erigibel, links deutlich.

2) Embolia cerebri. dextr.

F. S., weiblich, 27 Jahr. Patientin, seit einem halben Jahre (1874) linksseitig gelähmt, wird während des Aufenthaltes auf der Klinik bedeutend gebessert entlassen. Bauchreflex nicht geprüft, da ich auf denselben noch nicht achtete. September 1875 neuer Anfall, der wiederum völige linksseitige Lähmung bewirkt. 14 Tage später Bauchreflex links nicht nachweisbar, rechts deutlich. April 1876 derselbe Befund, Mamilla links nicht erigibel, rechts deutlich, sowie Contraction der Areola.

3) Hämorrhag cerebr. sinistri.

K. C., weiblich, 69 Jahr. Vollständige rechtsseitige Lähmung seit 10 Tagen. Bauchreflex fehlt rechts, links nur in der Seitenwand hervorzurufen. Ende April derselbe Befund. Mamilla und Areola reagiren links deutlich, rechts sehr langsam und schwach; die Contraction besteht aber rechts längel.

4) Hämorrhag. cerebr. dextr. ex nephritide interstitiali.

H., männlich, 24 Jahr. Vollständige linksseitige Hemiplegie, rorher öftere apoplectiforme Anfälle. Vor 8 Tagen ein Anfall, der zur Hemiplegie führte. Bauchreflex deutlich ausgesprochen rechts; fehlt links. Hodenveflex sehr deutlich rechts, fehlt links. Mamilla noch nicht geprüft. In Agone Bauchreflex fehlt links, rechts schwach. Hodenreflex links fehlend. Diagnose durch Section bestätigt.

5) S. F., männlich, $66 \mathrm{Jahr}$. Alte rechtsseitige Hirnhämorrhagie. Leichte linksseitige Parese zurückgeblieben. Patient kann Bewegungen beiderscits 
ast vollkommen gleich ausführen. Der Insult soll vor 16 Jahren stattgefunden haben. Bauchreflex beiderseits schwach, aber fast gleich. Kein Hodenreflex auf beiden Seiten. Mamilla noch nicht untersucht. Section ergab kleine alte Cysten in den motorischen Ganglien rechts neben allgemeiner Tuberculose.

6) H. V., männlich, $\boldsymbol{\tau}^{1} / 2 \mathrm{Jahr}$. Totale rechtsseitige Hemiplegie. (Pupillendifferenz, Facialislähmung und Lähmung der Extremitäten.) Die rechten Extremitäten sind verkürzt, atrophisch und namentlich der Arm ist functionsunfiahiger als links. Walrscheinlich Folge einer Hămorrhagie, die nach einer acuten Krankheit (Typhus) aufgetreten war. Bauchreflex rechts nur angedeutet, links sehr stark. Nach zwei Strichen verschwindet der Bauchreflex rechts, während er links stets hervorzurufen ist. Hodenreflex beiderseits deutlich und gleich stark.

7) Hämorrhag. cerebr. dextr. ex nephritid. interstitiali.

H, männlich, $38 \mathrm{Jahr}$. Totale linksseitigæ Hemiplegie; Coma. 4 Tage nach dem Insult fehlt der Bauchreflex links, rechts ist er sehr deutlich. Hodenreflex fehlt links, ist rechts zti constatiren. Zwölf Tage nach dem Anfall verschwindet das Coma. Die Reftexverhältnisse dieselben.

8) R., männlich, 52 Jahr. Wahrscheinlich Enbolie in Folge eines Klappenfehlers der Mitralis (Insufficienz vielleicht mit Stenose). Totale rechtsseitige Lähmung. Am zweiten Tage nach dem Insult Bauchreflex links sehr deutlich, fehlt rechts völlig. Hodenreflex konnte nicht ausgelöst werden. Mamilla reagirt links deutlich, rechts fast gar nicht; erst nach längerem Reize rechts eine schwache Contraction.*)

9) H. S., 22 Jahr. Hämorrhag. cerebr. dextr. (Für eine Embolie oder syphilitische Affection liegt kein Grund vor). Totale linksseitige Lähmung im November 1874. Im Mai 1876 noch deutliche Facialisparese links, Parese des linken Arms mit Atrophie, nur geringe Veränderungen der Juterextremität derselben Seite zu constatiren. Bauchreflex links nicht vorbanden, rechts sehr deutlich. Cremasterreflex links nicht zu constatiren, rechts ausgesprochen. Mamilla und Areola reagiren rechts sehr deutlich, links gar nicht oder sehr unbedeutend.

10) K. C., 64 Jahr. Encephalomalacia sin. Nephritis. Totale rechtsseitige Hemiplegie im Februar 1876. Im Mai 1876 ins Krankenhaus aufgenommen. Bauchreflex links schwach, rechts undeutlich vorhanden, Cremasterreflex beiderseits sehr deutlich, rechts etwas schwächer. Mamilla reagirt rechts sehr schwach, links deutlich.

Aus den beschriebenen Fällen scheint hervorzugehen, dass in frischen Fällen der Bauchreflex stets auf der gelähmten Seite fehlt, ebenso der Reflex von der Mamilla - doch sind über letztere meine Fälle noch zu wenig zahlreich - und dass das Ausbleiben des Cremasterreflexes ebenfalls ein constantes Symptom ist; eine Ausnahme scheint nur Fall 8 zu bilden. Ferner scheint, wie einige der vorstehenden Fälle beweisen, der Bauchreflex bei Hemiplegieen sehr lange zu fehlen, und erst nach mehrmonatlicher Dauer der

*) Fall 8 und 9 verdanke ich der Freundlichkeit des Herrn Dr. Th. Starck, 
Affection wieder aufzutreten, auch scheint er länger abgeschwächt zu sein als der Cremasterreflex. In einem der vorgeführten Fälle verschwand der Bauchreflex in der Agone; doch ist das Verschwinden dadurch erklärlich, dass das Abdomen bei der Respiration stark eingezogen warde und somit die Wahrnehmung des Reflexes erschwert war. Endlich will ich noch erwähnen, dass bei dem sub 6) erwähnten Knaben während des Schlafes alle Reflexe fehlten. Bei anderen Kranken gelang mir eine Prüfung der Reflexe im Schlafe nicht. Als Beweis dafür, dass es sich bei dem beschriebenen Symptom um einen Reflex und nicht um directe Muskelreizung analog der Contraction des Pectoralis beim Aufklopfen mit dem Percussionshammer handelt, lassen sich wohl folgende Gründe anführen, 1) Die Art der Contraction, welche in einem kräftigen Zusammenziehen der Bauchmuskeln besteht, während bei den directen Muskelreizungen durch mechanische Irritamente sich nur einzelne Partien zusammenziehen, die direct von dem Reize getroffen sind. 2) Die Beschaffenheit der Reizung selbst, da Nadelstiche und leichtes Ueberfahren mit dem Finger deutliche Zusammenziehung bewirken. 3) spricht gegen eine directe Muskelreizung die Unmöglichkeit durch das dicke Fettpolster des Abdomens hindurch mit Streichen einen Muskel zu irritiren. 4) erfolgt ja auch durch leises Kitzeln eine Einziehung des Abdomens, ein Vorgang, der entschieden für einen Reflexmechanismus spricht. Endlich könnte man noch gegen den Einwand, dass es sich um willkürliche Contractionen der Musculatur, gleichsam eine Abwehrbewegung handle, anführen, dass einerseits die Bauchmusculatur der betreffenden Seite in den beschriebenen Fällen, wie gleich ausgeführ't werden wird, für den Willen unerregbar ist, und ferner, dass andererseits im Coma der Bauchreflex auf der gesunden Seite vorhanden ist, in Fällen also, in denen die Willensaction ausgeschlossen ist. Schliesslich spricht für einen Reflex die Thatsache, dass nach längerer Reizung durch Streichen über das Abdomen - (verschieden lange bei verschiedenen Individuen) - der Reflex verschwindet, und dass man einen gewissen Zeitraum verstreichen lassen muss, ehe es gelingt, das Phänomen wieder hervorzurufen, also eine deutliche Erschöpfbarkeit des Reflexes. Auffallend ist es, dass der von Westphal und Erb beschriebene Patellarreflex auf der gelähmten Seite fast ausnahmslos stärker ist als auf der gesunden, während Bauch- und Cremasterreflex, also Hautreflexe, verschwunden sind. Beide genannten Autoren haben übrigens auf diese Differenz zwischen dem von ihnen beschriebenen und den Hautreflexen aufmerksam gemacht. Die Bahnen, in denen die Reflexaction sich abspielt, sind wabrscheinlich: Ileohypogastricus und Ileo-inguinalis, sowie die Cutanei abdominis anteriores als sensible IJeitungsbahnen, Lenden- und Brustmark als Uebertragungsstelle und die Nervi abdominis interni (Intercostales) und der Nerv. Ileo-hypogastricus als motorische Bahnen.

Auf die theoretisch interessanten Momente hat Jastrowitz bereits aufmerksam gemach1 und ich will aus oben angeführten Gründen auf dieselben nicht näher eingehen. Jedenfalls ist es im höchsten Grade bemerkenswerth, dass bei Hirnleiden oder besser bei Intactsein der spinalen Reflexbahnen, Reflexe ausbleiben, und dass dieselben einseitig sistirt sind. Die von Jastrowitz aufgeworfene Frage, ob der Bluterguss analog peripheren Reizen eine Hemmung der Reflexe im Rückenmark bewirken könne, Jässt sich vielleicht durch die Thatsache im negativen Sinne beantworten, dass es mir durch starke peri- 
phere Reize - Nadelstiche, electrischen Pinsel etc. - nicht gelungen ist, die Bauchreflexe bei Gesunden zu sistiren.

Schwierig ist die Entscheidung darüber, ob das Ausbleiben der Reflexe, sowohl des Cremaster- als des Bauchreflexes auf eine Lähmung der Bauchmusculatur, des Transversus und Obliquus abdominis zu beziehen ist, wie Jastrowitz es annimmt. Aus der Thatsache, dass ein Reflex verschwindet, lässt sich nicht sicher schliessen, dass der zur Reflexaction nothwendige Muskel afticirt ist, selbst dann nicht, wenn Integrität der sensiblen Bahnen angenommen werden kann. Wir können das Ausbleiben der geschilderten Reflexe durchaus nicht als stringenten Beweis - weder für Cremasterparalyse noch für Bauchmuskellähmung ansehen, sondern können eben nur den Vorgang, der sich bei Hemiplegischen in Bezug auf diese Reflexe abspielt, in das Gebiet der Reflexhemmungen verweisen. Viel wichtiger und beweisender für die Lähmung der Bauchmuskeln ist das andere von $J$ as trowitz angegebene Moment, nämlich das Untenbleiben des Hodens bei tiefen Inspirationen, vorausgesetzt, dass diese Inspirationen nicht reflectorischer Natur, sondern, dass sie vom Willen beeinflusst sind. Nur wenn in diesen Fällen der Hoden der gelähmten Seite nicht in die Höhe st igt, kann man auf Lähmung der Bauchmusculatur schliessen. Dass aber wirklich eine Lähmung der Rumpfmusculatur existirt, dafür spricht neben anderen später anzuführenden Thatsachen die Angabe von Nothnage I*), dass „schon bei ruhiger, sicher aber bei tiefer Respiration die Brusthälfte der gelähmten Seite sich im oberen Tiefen- und unteren Querdurchmesser weniger ausdehnt, als die andere (selbstverständlich ohne Affection der Lunge oder Pleura). Dies hängt von einer Parese der auxiliären Athemmuskeln ab, wie die Betastung der Scaleni lehrt. Ich betone diese betheiligung der Runjpfmusculatur ausdrücklich, weil ger:ıde diese der gewöhnlichen Anuahme nach meist frei bleiben soll." IIierher gehört auch die Angabe von Berger ${ }^{* *}$ ), dass ofter bei halbseitigen Lähmungen in Folge von Gehirnblutungen Paresen des Levator anguli scapulae und des Trapezius zu constatiren sind. Auch die Mittheilungen von Jastrowitz***) über die Lage der Kranken, ihr Herausrutschen aus dem Bette nach der gesunden Seite, die Schwierigkeit, sich sitzend zu erhalten, muss hierher bezogen werden, und scheint die von ihm gegebene Erklarung dicser Motilitätsstörungen als Affectionen der Rumpfimusculatur wohl acceptabel.

Ich möchte noch zum Schlusse bemerken, dass man an der Scapula be: tiefem Athmen namentlich deutlich die Differenz beider Seiten erkennen kann, indem die Weite des Interscapularraums sich auf der kranken Seite gar nicht oder fast nicht andert, da die Scapula der erkrankten Seite sich bei der Athmung gar nicht bewegt, eine Angabe, die mit den vorher angeführten zusammentrifft. Ebenso kann man, wenn man die Kranken pressen oder tief athmen lässt, an den oberen Theilen der Bauchmuskeln der gelähmten Scite ein Zurückbleiben in der Wölbung meist sehen, sicher aber eine geringere Resistenz fühlen, und das Zurückbleiben der betreffenden Seite palpatorisch constatiren.

*) Ziemssen, Handbuch der spec. Pathologie. Band XI. I. S. 96.

**) Die Lähmung des Nerv. thorac. longus.

***) L. c. 
Ein Beitrag zur Symptomatologic cerebraler Hemiplegieen.

Es ist zu wbinschen, dass zahlreiche Beobachtungen taber diese interessanten Verhältnisse, namentlich über das Vorkommen oder Verschwinden der Reflexe Aufschluss geben.

Schliesslich erlaube ich mir Herrn Professor Nothnagel für die bereitwillige Ueberlassung des Materials żu meinen Untersuchungen meinen besten Dank zu sagen.

Jena, im Mai 1876. 